\title{
Automatic Generation of Partial Order Set Hasse Diagram
}

\author{
Weicheng WANG ${ }^{1, a}$, shengling WANG ${ }^{2, b}$ \\ ${ }^{1}$. 2 College of Information Science and Technology \\ Beijing Normal University, Beijing 100875, China \\ a201411212045@mail.bnu.edu.cn, b wangshengling@bnu.edu.cn
}

key words: partial order set; Hasse diagram; automatic generation

\begin{abstract}
From the perspective of human-computer interaction, we design and implement a software that automatically generated frame graphics, which can conduct partial detection of any relationship, and then determine whether the poset is a lattice. By introducing the concept of Hasse diagram logic level and calculating the covering relation by poset, we can see the automatic generation of Hasse diagram on the screen, fully meet the consistency of theory and practice.
\end{abstract}

\section{Classification number: ТP391.6}

In computer science, the concept of relationship is of great importance. The set and the partial order relation defined on it form a set of partial positives. Partial relation is a typical and important relationship in relation, which is a transfer relation on a set, and provides a tool for comparing the order of elements. The partial order of Hasse diagram vividly describes the order relation between sets of elements, and simple reflects a relationship between elements of posets, which is a concise and effective representation of the diagram ${ }^{[1,2,3]}$.

The man-machine system is composed of computer, teachers and students in three aspects, formed a kind of automation teaching mode, using computer to display the contents of the curriculum for learners, completing the teaching function through the interaction between the learner and the computer.

It also changes the situation that the classroom teaching is the main and the students passively accept, and can simultaneously carry out individual teaching of many students. The use of modern education means to achieve the students as the center of the open individual education, ease the unity of the teaching content, teaching methods and the individual differences between students. A basic point of making full use of computer technology is the combination of graphics and knowledge base, in order to intuitively express the teaching information, improve teaching methods, produce an ideal environment, so that students learn a variety of knowledge through interactive learning. The Hasse diagram, which is designed and implemented here, can be used to generate the Hasse diagram of the partial set quickly, and effectively complete the generation of partial order relationship, covering relation, Hasse diagram and lattice series, promote the study and research on the theory and algorithm of Hasse diagram ${ }^{[4,5]}$.

\section{RELATION INPUT AND PARTIAL DECISION}

The relationship is a subset of the Cartesian product, that is, $R \subseteq X \times X$, where $\mathrm{X}=\{1,2, \ldots, \mathrm{n}\}, \mathrm{X}$ is general, and other types of sets can be bijective mapping of $X$. And the relationship can be further expressed as a relationship matrix $M$. It is natural to use this matrix as the data structure of the relationship, and when entering, each matrix element $m_{i j}$ is entered line by line. When $<i, j>\in R$, $m_{i j}=1$; when $<i, j>\notin R, m_{i j}=0$.

Whether the input relationship is a partial order is to determine whether it has reflexive, anti-symmetric, transitive. According to the given relation matrix $\mathrm{M}$, if the main diagonal of $\mathrm{M}$ is "1", the relation $\mathrm{R}$ is reflexive.

If for any $\mathrm{i}, \mathrm{j}(\mathrm{i} \neq \mathrm{j}, \mathrm{i} \leq \mathrm{n}, \mathrm{j} \leq \mathrm{n})$, there is $m_{i j} * m_{i j}=0$, then $\mathrm{R}$ is anti-symmetric. Defined by the transitivity, if the relation $\mathrm{R}$ with the transmission, then there must be $m_{i k}=1 \wedge m_{k j}=1 \Rightarrow m_{i j}=1$, the formula can be rewritten as inverse, $m_{i j}=0 \Rightarrow m_{i k}=0 \vee m_{k j}=0$, which can determine the transfer of R. This can be described by the following algorithm1.

\section{Algorithm 1 Determine the partial order}


(1) Input the relation matrix $M$ of relaiton $R$, which is of order $n$;

(2) Set the flag variable $T=1$;

(3) To determine the reflexivity, for $i=1,2, \ldots, n$, if there is $m_{i i}=0$, then $\mathrm{R}$ is non-reflexive, set $T=0$, turn (16);

(4) To Determine the anti-symmetric, for $i=2,3, \ldots, n$; $\mathrm{j}=1,2, \ldots \mathrm{I}-1$, if there is $m_{i j} * m_{j i}=1$, then $R$ is not anti-symmetric, set $\mathrm{T}=0$, turn (16);

(5) Line initial value $i=1$, and start transitivity judgment;

(6) If $i>n$, turn (16); matrix decision is completed;

(7) Column initial variable $j=1$;

(8) If $j>n$, turn (15); the judgment for the line is completed;

(9) If $m_{i j}=1$,turn (14);

(10) Set the variable $k=1$;

(11) If $k>n$, turn (14);

(12) If $m_{i k}=m_{k j}=1$, then $R$ is non-transitive, set $T=0$, turn (16);

(13) $k$ shows an increase of 1 , turn (11);

$j$ shows an increase of 1 , turn (8); Column progressively increase;

(14) $i$ shows an increase of 1, turn (6); line progressively increase;

(15) If $T=1$, then $R$ is a partial order relation, otherwise $R$ is not a partial order relation;

(16) End.

\section{THE JUDGMENT OF THE LATTICE}

Lattice is the further deepening of the concept of partial order, which it has important applications in cryptography and switching theory. The key is to determine whether the arbitrary two elements in $\mathrm{X}$ have supremum and infimum by the partial order relation $\mathrm{R}$ on $\mathrm{X}$.

The decision of the lattice can also be done by means of a relation matrix, which can be considered from the following aspects:

(1) Since R is reflexive, for two of the same elements in X, such as $a, a$,

(2) There must be $\langle a, a\rangle_{\in R}$, that is, there is partial order relationship between a and a, the two same elements have the supremum and infimum which are their own.

(3) Let's scan the upper triangular elements of the relation matrix line by line.

When $m_{i j}=1 \quad(i \neq j)$, it is shown that there is a partial order relation between the two elements a and $\mathrm{b}$ in the corresponding order $<\mathrm{a}, \mathrm{b}>$, one is supremum and the other is infimum.

When $m_{i j}=0(i \neq j)$, if $m_{i j}=1$, in fact it can be considered together with the above situation; if $m_{i j}$ $=0$, we do the following treatment:

(1) We scan the i-th row and the j-th row to find all the k satisfying $m_{i k}=m_{j k}=1$

We may set up a collection of elements corresponding to these $k$ be $C=\left\{c_{1}, \ldots, c_{p}\right\}$. Then $\mathrm{C}$, that is, $m_{i j}$ corresponds to the upper bound of element $a, b$.

(2) Simultaneous scanning $c_{1}, \ldots, c_{p}$ each column, but we only consider the line $c_{1}, \ldots, c_{p}$

If there is $c_{i}$ to make $m_{c_{i} c_{1}}=\ldots=m_{c_{i} c p}=1$, then there is a minimal element $c_{i}$ in the set $\mathrm{C}$, which is the supremum of the element $a, b$.

(3) Scan the i-th row and the $\mathrm{j}$-th row to find all the k satisfying $m_{i k}=m_{j k}=1$

We may set up a collection of elements corresponding to these $k$ be $D=\left\{d_{1}, \ldots, d_{q}\right\}$. Then $D$, that is, $m_{i j}$ corresponds to the lower bound of element $a, b$.

(4) Simultaneous scanning $d_{1}, \ldots, d_{q}$ each column

We only consider the line $d_{1}, \ldots, d_{q}$. If there is $d_{i}$ to make $m_{c_{i} c_{1}}=\ldots=m_{c_{i} c p}=1$, then there is a maximal element $d_{i}$ in the set $D$, which is the infimum of the element $a, b$. The process can be represented in Fig.1 . 


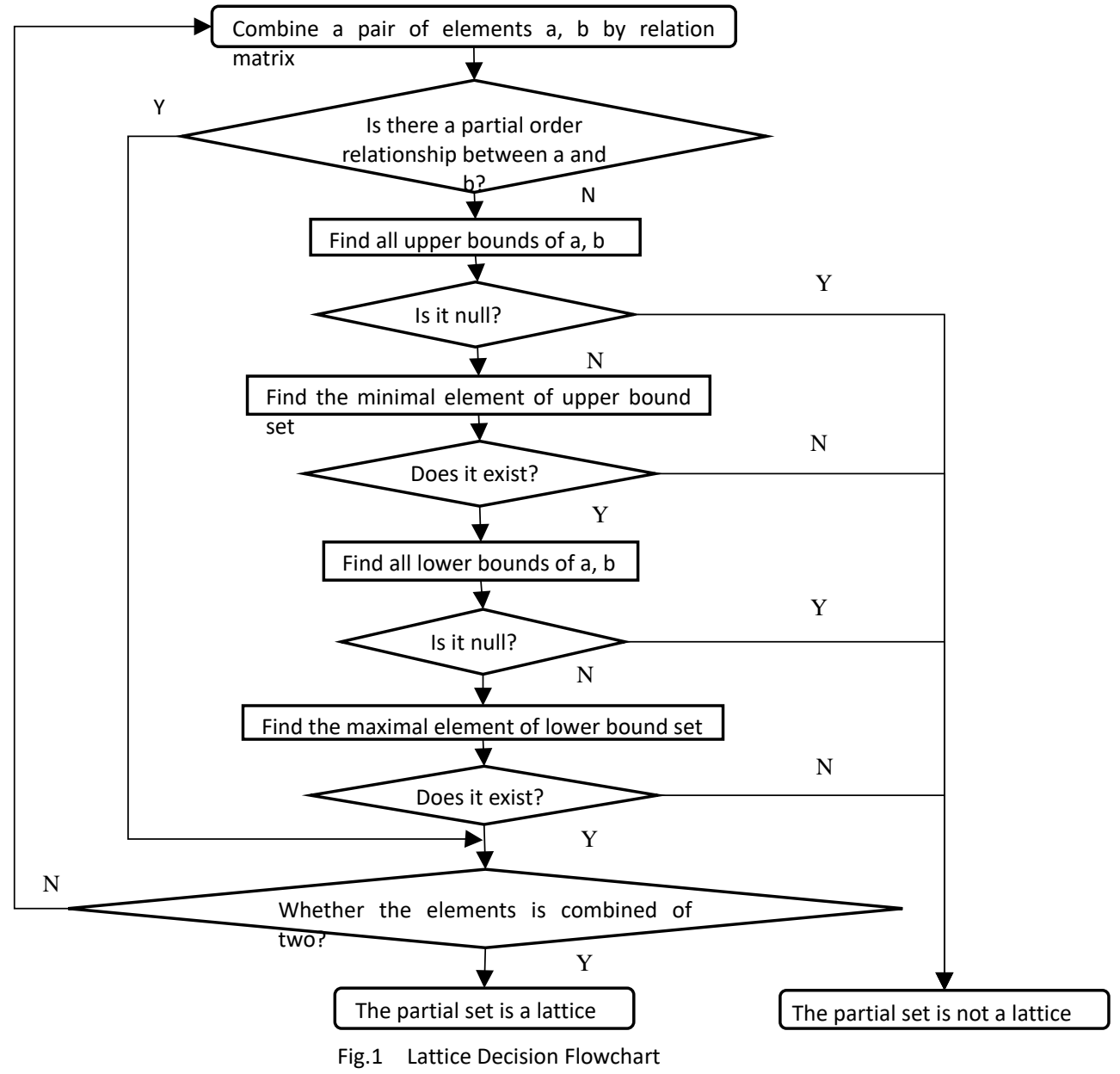

\section{THE GENERATION OF THE OVERLAY RELATIONSHIP}

The overlay relationship is a bridge that expresses the partial order into a Hasse diagram, and the Hasse diagram can be made with the property of the overlay. The overlay relationship can also be expressed as a matrix, as long as the relationship matrix can be transformed into a overlay matrix through rules. The algorithm 2 describes the basic idea.

Algorithm 2 Seek the overlay relationship

(1) Copy the relationship matrix $M$ to $C$;

(2) The element value on the main diagonal of the $C$ matrix is 0 , and the reflexivity is eliminated;

(3) Scan matrix $C$ by row and column;

If there is an element $C[i, j]=0$, then no treatment, take the next element;

If the there is an element $C[i, j]=1$, you must determine whether there is a transitive relationship between $\mathrm{i}$ and $\mathrm{j}$, as follows:

(1) Set $k=1$;

(2) If $C[i, k]=C[k, j]=1$, there is transfer between $i$ and $j$, set $C[i, j]=0, k=n$;

(3) $k+1 \Rightarrow k$;

(4) If $k \leq n$, turn (2);

(5) End.

\section{AUTOMATIC GENERATION OF HASSE DIAGRAM}

A distinctive feature of Hasse diagram is that it reflects the hierarchical relationship between elements in the partial order set $<\mathrm{X}, \mathrm{R}>$ (poset). Therefore, to draw a complete Hasse diagram must determine the level of all elements, which can be achieved by overlay matrix. It is described by algorithm 3.

Algorithm 3 Hierarchical relationship of Hasse diagram

Initialize $D[1 \ldots n]=0$; Copy the cover matrix into the same dimension matrix $A$; 
WHILE $F=1$

Set $F=0$

FOR $i=1$ To $n$

IF $D[i]=0 \quad$ Scan the $i$-th row of $A$;

If all is 0 , set $D[i]=P, F=1$;

ENDIF; \{Determine if $i$ is on $P$-th layer

ENDFOR; $\{$ The elements of $P$-th layer is determined $\}$

FOR $j=1$ To $n$

IF $D[i]=P$ The $j$-th row of matrix A is always zero; END IF;

END FOR; $\{$ Eliminate the influence of P-th layer elements

$P=P+1 ; \quad\{$ level increased by 1 ; determine the next layer $\}$

END WHILE.

After the algorithm is completed, the matrix $D$ stores the levels of the elements in the Hasse diagram.

With the overlay relationship and hierarchical relationship, we can generate Hasse diagram more easily. We can first open up a memory buffer, in the buffer to make Hasse diagram, and then quickly displayed from the screen or output through the printer.

In the process of drawing, the D matrix is used to determine the level of each node. We first output the small level and put it at the top, then output of each node layer by layer, and control the spacing between the nodes and column to make the appearance of the Hasse diagram beautiful. This process is complicated. Then, according to the information provided by the overlay matrix $C$, a straight line is connected between all the elements that have overlap relationship, so that a complete Hasse diagram is made in the buffer. Finally, we turn the Hasse diagram in the buffer into a dot array in screen or use dot-matrix printer to obtain Hasse diagram. When the Hasse diagram size exceeds the size of a screen, it provides a window upper and down, left and right movement and page functions to view the Hasse diagram of the various parts, while the Hasse diagram can be saved to the file. When necessary, it can be directly transferred out at any time. The software functional is very flexible and convenient.

In the realization, with $\mathrm{C}++$ programming successfully achieved this software, learners can personally hands on the operation of the practice, learning partial set, lattice structure, Hasse diagram and other related professional content, so as to achieve a deeper understanding of the concept, to promote image thinking, learning efficiency and learning interest. What's more, the software will also promote the relevant scientific research.

\section{ACKNOWLEDGMENT}

The Project Supported by National Natural Science Foundation of China, Grant NO.61472044

\section{REFERENCES}

[1] Fang Shican. Discrete Mathematics[M]. Xidian University Press, 2010, 109 115

[2] Zou Youjiao, Ran Zhanjun, Wang Xiaofeng. A Solution of Hasse Diagram with Partial-order Relation[J]. Studies in College Mathematics， 2013， 16(1): 55 58

[3] Ding Shuliang, Luo Fen. Algorithm:From Poset to Hasse Diagram[J]. Studies in College Mathematics, 2005, 29(2): 150 153

[4] Pan Meiqing, Ding Zhijun. An Algorithm of quick Evaluating Hasse diagram[J]. Journal of Shandong University of Science and Technology, 2013，22(3): 88 90

[5] Bernard Kolman, etal. Discrete Mathematical Structures[M]. High Education Press, 2001

\section{AUTHOR INTRODUCTION}

Weicheng Wang, Born in 1996, Male, Bachelor. College of Information Science and Technology, Beijing Normal University, Beijing 100875, China.

Shengling Wang, Born in 1975, Female, Ph.D, Professor. College of Information Science and Technology, Beijing Normal University, Beijing 100875, China. 